\title{
FRANÇOIS GRAMAIN
}

\section{Transcendance et fonctions modulaires}

Journal de Théorie des Nombres de Bordeaux, tome 11, no 1 (1999), p. $73-90$

<http://www.numdam.org/item?id=JTNB_1999_11_1_73_0>

(C) Université Bordeaux 1, 1999, tous droits réservés.

L'accès aux archives de la revue « Journal de Théorie des Nombres de Bordeaux » (http://jtnb.cedram.org/) implique l'accord avec les conditions générales d'utilisation (http://www.numdam.org/conditions). Toute utilisation commerciale ou impression systématique est constitutive d'une infraction pénale. Toute copie ou impression de ce fichier doit contenir la présente mention de copyright.

\section{Numdam}

Article numérisé dans le cadre du programme

Numérisation de documents anciens mathématiques

http://www.numdam.org/ 
Journal de Théorie des Nombres

de Bordeaux 11 (1999), 73-90

\title{
Transcendance et fonctions modulaires
}

\author{
par FRANÇOIS GRAMAIN
}

\begin{abstract}
RÉSUMÉ. Cette rédaction contient l'exposé fait aux Journées Arithmétiques 97 et une annexe concernant la méthode de Mahler. Le début de l'exposé présente les notions mises en jeu dans le cœur du sujet (courbes elliptiques et formes modulaires). Pour un traitement complet de ces notions on peut se référer à [Ser], [Lan1] et [Lan2]. Une preuve complète du théorème de Yuri Nesterenko se trouve, en dehors de l'article original ([Nes1] pour l'annonce et [Nes2] pour les démonstrations), dans les exposés de Michel Waldschmidt au Séminaire Bourbaki [Wal1] et à Carleton [Wal2]. Pour les résultats quantitatifs les plus récents, on pourra consulter [Nes3].
\end{abstract}

ABSTRACT. English title: Transcendence and modular functions. After recalling links between elliptic curves and modular functions, we present, with numerous corollaries (in particular the algebraic independence of $\pi, e^{\pi}$ and $\Gamma(1 / 4)$ ), the theorem obtained by Yuri Nesterenko in 1996: if $E_{2}, E_{4}$ and $E_{6}$ are the three first Eisenstein series and if $q$ satisfies $0<|q|<1$ (in both complex and $p$-adic cases $)$, then the field $\mathbb{Q}\left(q, E_{2}(q), E_{4}(q), E_{6}(q)\right)$ has transcendence degree at least 3 over $\mathbb{Q}$. The origin of Nesterenko's work is the proof, in 1995 by a team of Saint-Etienne, of MahlerManin conjecture about the modular invariant $J=1728 \frac{E_{4}^{3}}{E_{4}^{3}-E_{6}^{2}}$ : if $q \in \mathbb{C}$ or $q \in \mathbb{C}_{p}$ satisfies $0<|q|<1$, then $q$ and $J(q)$ are not both algebraic. An annex describes the transcendence method of Mahler and why it does not work in this case.

\section{INTRODUCTION HISTORIQUE}

L'étude du polynôme modulaire permet de prouver que si $\tau$ est un nombre algébrique de degré 2 , alors la valeur $j(\tau)$ de l'invariant modulaire est aussi un nombre algébrique. Ce résultat admet une réciproque :

Théorème (Schneider 1937). Quand $\tau$ est algébrique, la valeur $j(\tau)$ de l'invariant modulaire est algébrique si et seulement si $\tau$ est quadratique.

La preuve utilise les fonctions elliptiques de Weierstrass et Theodor Schneider a posé dans son livre [Sch] le problème (deuxième problème de 
Schneider) de démontrer ce théorème par une étude directe de la fonction $j$, et non par l'étude des fonctions de Weierstrass. Ce problème est toujours ouvert, mais une question voisine, posée par Kurt Mahler en 1969 (voir [Mah1], mais K. Mahler posait déjà cette question à ses étudiants en 1964), a été résolue en 1995 [BDGP] par une méthode n'utilisant que les propriétés de modularité de $j$ :

Théorème stéphanois. Si $q \in\{z \in \mathbb{C} ; 0<|z|<1\}$ est algébrique, alors $J(q)$ est transcendant.

Ici, comme dans tout l'exposé, la fonction $J$ est définie par $j(\tau)=$ $J\left(e^{2 i \pi \tau}\right)$. La démonstration, une adaptation de la méthode de Mahler qui est présentée en annexe, s'applique pour donner le résultat analogue dans le cas $p$-adique (conjecturé en 1971 par Yu. V. Manin [Man]). Plus généralement, presque tous les résultats cités dans ce texte ont des analogues $p$-adiques, mais nous ne parlerons que du cas complexe.

Dans toutes ces questions de transcendance ou d'indépendance algébrique, le point de vue modulaire et le point de vue elliptique sont intimement liés. Je crois que Daniel Bertrand a été le premier (dans les années 70) à utiliser systématiquement ces liens pour obtenir de nouveaux résultats et poser les bonnes questions.

\section{UN DICTIONNAIRE}

Soit $L=\mathbb{Z} \omega_{1}+\mathbb{Z} \omega_{2}$ le réseau de $\mathbb{C}$ engendré par les nombres complexes $\omega_{1}$ et $\omega_{2}$ linéairement indépendants sur $\mathbb{R}$. Le groupe quotient $\mathbb{C} / L$ est ce qu'on appelle une courbe elliptique.

$\operatorname{Si} \wp(z)=\frac{1}{z^{2}}+\sum_{\substack{\omega \in L \\ \omega \neq 0}}\left(\frac{1}{(z-\omega)^{2}}-\frac{1}{\omega^{2}}\right)$ est la fonction de Weierstrass associée au réseau $L$, l'application

$$
\begin{aligned}
\mathbb{C} & \longrightarrow \mathbb{P}_{2}(\mathbb{C}) \\
z \notin L & \longmapsto\left(1: \wp(z): \wp^{\prime}(z)\right) \\
\omega \in L & \longmapsto(0: 0: 1)
\end{aligned}
$$

induit un isomorphisme analytique

$$
\mathbb{C} / L \simeq \mathcal{E}_{L},
$$

où $\mathcal{E}_{L}$ est la courbe d'équation affine

$$
y^{2}=4 x^{3}-g_{2}(L) x-g_{3}(L)
$$

et les invariants $g_{2}(L)$ et $g_{3}(L)$ de $\mathcal{E}_{L}$ sont donnés par

$$
g_{2}(L)=60 \sum_{\substack{\omega \in L \\ \omega \neq 0}} \frac{1}{\omega^{4}} \quad \text { et } \quad g_{3}(L)=140 \sum_{\substack{\omega \in L \\ \omega \neq 0}} \frac{1}{\omega^{6}} .
$$


Deux courbes elliptiques $\mathbb{C} / L$ et $\mathbb{C} / M$ sont analytiquement isomorphes si et seulement si les réseaux $L$ et $M$ sont homothétiques (i.e. s'il existe $\alpha \in \mathbb{C}^{\times}$ tel que $M=\alpha L$ ). L'isomorphisme se lit aussi sur les équations des courbes : les courbes elliptiques $\mathbb{C} / L$ et $\mathbb{C} / M$ sont analytiquement isomorphes si et seulement si $j(L)=j(M)$, où

$$
j(L)=1728 \frac{g_{2}^{3}(L)}{g_{2}^{3}(L)-27 g_{3}^{2}(L)} .
$$

Tout réseau peut s'écrire $\mathbb{Z} \omega_{1}+\mathbb{Z} \omega_{2}=\omega_{1}(\mathbb{Z}+\tau \mathbb{Z})$, avec $\tau \in \mathfrak{H}$ où

$$
\mathfrak{H}=\{\tau \in \mathbb{C} ; \operatorname{Im}(\tau)>0\},
$$

de sorte que l'invariant $j$ peut être vu comme une fonction

$$
j: \mathfrak{H} \longrightarrow \mathbb{C}
$$

qui, de plus, est holomorphe sur $\mathfrak{H}$.

Les relations $\mathbb{Z}+\tau \mathbb{Z}=\mathbb{Z}+(\tau+1) \mathbb{Z}=\tau\left(\mathbb{Z}+\frac{-1}{\tau} \mathbb{Z}\right)$ donnent immédiatement

$$
\begin{gathered}
j(\tau+1)=j(\tau) \text { et } j\left(\frac{-1}{\tau}\right)=j(\tau), \text { donc } \\
j\left(\frac{a \tau+b}{c \tau+d}\right)=j(\tau) \text { pour tout } \tau \in \mathfrak{H} \text { et tout }\left(\begin{array}{ll}
a & b \\
c & d
\end{array}\right) \in S L_{2}(\mathbb{Z}) .
\end{gathered}
$$

On dit que l'invariant $j$ est modulaire. Plus généralement, pour traiter le cas de $g_{2}$ et $g_{3}$ (que l'on peut voir comme définis sur $\mathfrak{H} \operatorname{par} g_{i}(\tau)=g_{i}(\mathbb{Z}+\tau \mathbb{Z})$ ), on définit la

\section{Modularité.}

Une fonction $f$ méromorphe sur le demi-plan supérieur

$$
\mathfrak{H}=\{\tau \in \mathbb{C} ; \operatorname{Im}(\tau)>0\}
$$

est dite faiblement modulaire de poids $2 k(k \in \mathbb{N})$ si et seulement si

$$
f\left(\frac{a \tau+b}{c \tau+d}\right)=(c \tau+d)^{2 k} f(\tau) \text { pour tout } \tau \in \mathfrak{H} \text { et tout }\left(\begin{array}{ll}
a & b \\
c & d
\end{array}\right) \in S L_{2}(\mathbb{Z}) .
$$

En particulier on a $f(\tau+1)=f(\tau)$, de sorte que $f$ possède un développement de Fourier à l'infini

$$
f(\tau)=F(z)=\sum_{n \in \mathbb{Z}} a_{n} z^{n},
$$

où $z=e^{2 i \pi \tau}$. Si la série $F$ a seulement un pôle en 0 (on dit que $f$ est méromorphe à l'infini), $f$ est une fonction modulaire.

L'invariant modulaire $j$ est l'unique (à une constante additive près) fonction modulaire de poids 0 ayant un pôle simple à l'infini de résidu 1 : 


$$
\begin{aligned}
j(\tau)=J(z) & =\frac{\left(1+240 \sum_{n \geq 1} n^{3} z^{n} /\left(1-z^{n}\right)\right)^{3}}{z\left(\prod_{n \geq 1}\left(1-z^{n}\right)\right)^{24}} \\
& =\frac{1}{z}+744+196884 z+21493760 z^{2}+\sum_{n \geq 3} c(n) z^{n}
\end{aligned}
$$

et les $c(n)$ sont des entiers positifs. On démontre que la série $J$ est convergente dans le disque unité pointé $\mathcal{D}=\{z \in \mathbb{C} ; 0<|z|<1\}$.

Une forme modulaire est une fonction modulaire qui est holomorphe sur $\mathfrak{H}$ et à l'infini ; dans ce cas la série $F$ est entière.

\section{Séries d'Eisenstein}

Si, pour $n \in \mathbb{N}$, on pose $\sigma_{k}(n)=\sum_{d \mid n} d^{k}$, alors, en notant $z=e^{2 i \pi \tau}$ où $\tau \in \mathfrak{H}$,

$P(z)=E_{2}(z)=1-24 \sum_{n \geq 1} \sigma_{1}(n) z^{n}$ ne définit pas une forme modulaire de poids 2 ; mais presque, comme le montre la formule

$$
\begin{gathered}
P\left(e^{-2 i \pi / \tau}\right)=\tau^{2} P\left(e^{2 i \pi \tau}\right)+\frac{6 \tau}{i \pi} \\
Q(z)=E_{4}(z)=1+240 \sum_{n \geq 1} \sigma_{3}(n) z^{n}=\frac{12}{(2 \pi)^{4}} g_{2}(\tau) \\
R(z)=E_{6}(z)=1-504 \sum_{n \geq 1} \sigma_{5}(n) z^{n}=\frac{216}{(2 \pi)^{6}} g_{3}(\tau)
\end{gathered}
$$

est une forme modulaire de poids 6 .

La formule donnant $j$ en fonction de $g_{2}$ et $g_{3}$ se traduit donc en

$$
J=1728 \frac{Q^{3}}{Q^{3}-R^{2}} .
$$

Le $\mathbb{C}$-espace vectoriel des formes modulaires est une algèbre graduée et il est engendré en tant qu'anneau par les formes $Q$ et $R$ :

$$
M=\coprod_{k \geq 0} M_{k}=\mathbb{C}[Q, R],
$$

où $M_{k}$ est l'espace vectoriel des formes modulaires de poids $2 k: M_{0}=\mathbb{C}$, $M_{1}=\{0\}, M_{2}=\mathbb{C} Q, M_{3}=\mathbb{C} R, \ldots, M_{6}=\mathbb{C} Q^{3}+\mathbb{C} R^{2} \ldots$

Le fait que l'opérateur différentiel $12 z \frac{d}{d z}-2 k P$ envoie $M_{k}$ dans $M_{k+1}$ est fondamental. C'est lui qui explique les relations de base liant les fonctions $P, Q$ et $R$ par l'intermédiaire de l'opérateur $\Theta=z \frac{d}{d z}=\frac{1}{2 i \pi} \frac{d}{d \tau}$ : 


$$
\Theta P=\frac{1}{12}\left(P^{2}-Q\right), \quad \Theta Q=\frac{1}{3}(P Q-R), \quad \Theta R=\frac{1}{2}\left(P R-Q^{2}\right) .
$$

Comme conséquence immédiate de ces relations on obtient

$$
\frac{\theta J}{J}=-\frac{R}{Q} \quad 6 \frac{\theta^{2} J}{\theta J}=P-\frac{4 R}{Q}-\frac{3 Q^{2}}{R}
$$

et les relations réciproques

$$
\begin{aligned}
P & =6 \frac{\theta^{2} J}{\theta J}-4 \frac{\theta J}{J}-3 \frac{\Theta J}{J-1728}, \\
Q & =\frac{(\Theta J)^{2}}{J(J-1728)}, \\
R & =\frac{-(\Theta J)^{3}}{J^{2}(J-1728)}
\end{aligned}
$$

de sorte que, en notant $f(z)$ la fonction $z \mapsto f(z)$, on a l'identité des corps

$$
\begin{aligned}
\mathbb{Q}(z, P(z), Q(z), R(z)) & =\mathbb{Q}\left(z, J(z), \Theta J(z), \Theta^{2} J(z)\right) \\
& =\mathbb{Q}\left(z, J(z), J^{\prime}(z), J^{\prime \prime}(z)\right) .
\end{aligned}
$$

Quand on passe des fonctions aux valeurs qu'elles prennent en un point, les facteurs $J$ et $J-1728$ en dénominateur obligent à exclure quelques points : pour $\tau \not \equiv i$ et $\tau \not \equiv \rho=e^{2 i \pi / 3} \bmod S L_{2}(\mathbb{Z})$, les corps engendrés sur $\mathbb{Q}$ par les nombres $q=e^{2 i \pi \tau}, P(q), Q(q)$ et $R(q)$ ou par $e^{2 i \pi \tau}, j(\tau), j^{\prime}(\tau) / \pi$ et $j^{\prime \prime}(\tau) / \pi^{2}$ ont le même degré de transcendance sur $\mathbb{Q}$.

On complète le dictionnaire par les relations suivantes : soient $\wp$ la fonction elliptique de Weierstrass associée au réseau $L=\mathbb{Z} \omega_{1}+\mathbb{Z} \omega_{2}$, où $\tau=\frac{\omega_{2}}{\omega_{1}} \in \mathfrak{H}$, et $\eta_{1}$ la quasi-période associée à $\omega_{1}$ (la quasi-période associée à la période $\omega$ de la fonction $\wp$ est définie par $\eta=\zeta(z+\omega)-\zeta(z)$, où $\zeta^{\prime}=-\wp$ ), alors on a :

$$
\begin{aligned}
& P(q)=3 \frac{\omega_{1}}{\pi} \frac{\eta_{1}}{\pi}, \\
& Q(q)=\frac{3}{4}\left(\frac{\omega_{1}}{\pi}\right)^{4} g_{2}(L), \\
& R(q)=\frac{27}{8}\left(\frac{\omega_{1}}{\pi}\right)^{6} g_{3}(L) .
\end{aligned}
$$

En utilisant ce dictionnaire, le résultat suivant (dû à Th. Schneider) Si $\omega \neq 0$ est une période d'une fonction $\wp$ de Weierstrass d'invariants algébriques $g_{2}$ et $g_{3}$, alors $\frac{\omega}{\pi}$ est un nombre transcendant se traduit en

Pour $q \in \mathcal{D}$ les nombres $Q(q)$ et $R(q)$ ne sont pas tous deux algébriques ou en 
Pour $q \in \mathcal{D}$ et $J(q) \notin\{0,1728\}$ les nombres $J(q)$ et $q J^{\prime}(q)$ ne sont pas simultanément algébriques.

En effet, on obtient un énoncé équivalent au premier énoncé en y remplaçant $\omega$ par $\omega_{1}$ (une période primitive). Pour le voir, il suffit de remplacer le réseau des périodes de $\wp$ par un réseau homothétique dans un rapport entier, ce qui, par homogénéité des fonctions $g_{2}$ et $g_{3}$ ne change rien aux propriétés de transcendance. Le deuxième énoncé est trivial si $J(q)$ est transcendant. Si $J(q)$ est algébrique, on choisit un réseau $L$ d'invariants $g_{2}$ et $g_{3}$ algébriques tels que $J(q)=1728 \frac{g_{2}^{3}}{g_{2}^{3}-27 g_{3}^{2}}$ et les dernières formules citées donnent l'équivalence des deux énoncés. Le troisième énoncé et le second se déduisent l'un de l'autre par les formules liant les séries d'Eisenstein à $J$ et $\Theta J$.

C'est cette traduction qui a inspiré D. Bertrand [Ber1] en 1975 pour obtenir l'analogue $p$-adique du résultat de Th. Schneider en utilisant les fonctions elliptiques de Jacobi-Tate au lieu des fonctions de Weierstrass. Katia Barré [Bar1] a obtenu en 1995 le même résultat dans les cas complexe et $p$-adique par une preuve purement modulaire.

De la même façon, ce résultat de Gregory Chudnovsky (1977, voir [Chu]) Si $\eta$ est la quasi-période associée à la période $\omega \neq 0$ de la fonction elliptique $\wp$ de Weierstrass, alors $\operatorname{degtr}_{\mathbb{Q}} \mathbb{Q}\left(g_{2}, g_{3}, \frac{\omega}{\pi}, \frac{\eta}{\omega}\right) \geq 2$

se traduit en

Pour $q \in \mathcal{D}$ on $a \operatorname{degtr}_{\mathbb{Q}} \mathbb{Q}(P(q), Q(q), R(q)) \geq 2$.

Là aussi, D. Bertrand a exploité les traductions de cet énoncé et il conjecturait dans son article de 1978 [Ber2] le résultat suivant (dans le cas où $q$ est algébrique) :

\section{Le Théorème de Yuri Nesterenko}

Théorème (Yu. Nesterenko 1996). Pour $q \in \mathcal{D}$ on a

$$
\operatorname{degtr}_{\mathbb{Q}} \mathbb{Q}(q, P(q), Q(q), R(q)) \geq 3 .
$$

Notre dictionnaire fournit une quantité de traductions ou de conséquences de ce résultat, par exemple :

Si $\tau \not \equiv i, \rho \bmod S L_{2}(\mathbb{Z})$, alors $\operatorname{degtr}_{\mathbb{Q}} \mathbb{Q}\left(q, J(q), J^{\prime}(q), J^{\prime \prime}(q)\right) \geq 3$ et $\operatorname{degtr}_{\mathbb{Q}} \mathbb{Q}\left(e^{2 i \pi \tau}, j(\tau), j^{\prime}(\tau) / \pi, j^{\prime \prime}(\tau) / \pi^{2}\right) \geq 3$.

Si $q \in \mathcal{D}$ est algébrique (dans ce cas $\tau$ est transcendant d'après le théorème de Gel'fond-Schneider), alors $P(q), Q(q)$ et $R(q)$ sont algébriquement indépendants sur $\mathbb{Q}$, de même que $J(q), J^{\prime}(q)$ et $J^{\prime \prime}(q)$. 
$S i \wp$ est une fonction elliptique d'invariants $g_{2}$ et $g_{3}$ algébriques, alors $e^{2 i \pi \tau}, \frac{\omega_{1}}{\pi}$ et $\frac{\eta_{1}}{\pi}$ sont algébriquement indépendants sur $\mathbb{Q}$.

Dans le cas de la multiplication complexe (c'est-à-dire lorsque $\tau$ est un nombre algébrique quadratique), grâce aux relations de Legendre et de David Masser ([Mas], lemma 3.1 et appendix 1), on obtient :

$S i \wp$ est une fonction elliptique d'invariants $g_{2}$ et $g_{3}$ algébriques et à multiplication complexe, chacun des deux triplets $\left\{e^{2 i \pi \tau}, \omega, \eta\right\}$ et $\left\{e^{2 i \pi \tau}, \omega, \pi\right\}$ est constitué de nombres algébriquement indépendants sur $\mathbb{Q}$.

En effet, en notant $\eta$ la quasi-période associée à la période $\omega$, la relation de Legendre s'écrit $\eta_{1} \omega_{2}-\eta_{2} \omega_{1}=2 i \pi$. Si, de plus, on est dans le cas de multiplication complexe et si l'équation minimale de $\tau$ est $A+B \tau+C \tau^{2}=0$, la relation de D. Masser est $A \eta_{1}-C \tau \eta_{2}=\kappa \omega_{2}$, où $\kappa$ est un nombre algébrique. Pour démontrer le résultat annoncé, une homothétie entière sur le réseau des périodes permet, comme plus haut, de supposer que $\omega=\omega_{1}$. Il suffit alors de démontrer que $\pi$ est algébrique sur $\mathbb{Q}\left(\omega_{1}, \eta_{1}\right)$ et que $\eta_{1}$ est algébrique sur $\mathbb{Q}\left(\pi, \omega_{1}\right)$.

Démontrons la première assertion : comme $\omega_{2}=\tau \omega_{1}$ est algébrique sur $\mathbb{Q}\left(\omega_{1}, \eta_{1}\right)$, la relation de D. Masser montre que $\eta_{2}$ est algébrique sur $\mathbb{Q}\left(\omega_{1}, \eta_{1}\right)$, puisque $C \neq 0$ (car $\tau$ n'est pas réel).

Pour prouver la seconde assertion, on élimine $\eta_{2}$ entre les relations de Legendre et D. Masser, ce qui donne la relation $\left(A \omega_{1}-C \tau \omega_{2}\right) \eta_{1}=\kappa \omega_{1} \omega_{2}-$ $2 i \pi C \tau$. Mais $\omega_{2}=\tau \omega_{1}$, donc on a $\left(A-C \tau^{2}\right) \omega_{1} \eta_{1}=\kappa \tau \omega_{1}^{2}-2 i \pi C \tau$. Il suffit donc de voir que $A-C \tau^{2} \neq 0$ pour conclure. Or cela résulte du fait que $C$ n'est pas nul et que le polynôme minimal de $\tau$ est $A+B X+C X^{2}$. En particulier, pour $\tau=i$ (resp. $\tau=\rho$ ) la courbe elliptique d'équation $y^{2}=4 x^{3}-4 x\left(\right.$ resp. $\left.y^{2}=4 x^{3}-4\right)$ donne $\omega=\frac{\Gamma(1 / 4)^{2}}{\sqrt{8 \pi}}($ resp. $\omega=$ $\left.\frac{\Gamma(1 / 3)^{3}}{2^{4 / 3} \pi}\right)$, de sorte que :

Les trois nombres $\pi$, $e^{\pi}$ et $\Gamma(1 / 4)$ sont algébriquement indépendants sur $\mathbb{Q}$, de même que $\pi, e^{\pi \sqrt{3}}$ et $\Gamma(1 / 3)$.

Ainsi un vieux rêve est réalisé :

\section{$\pi$ et $e^{\pi}$ sont algébriquement indépendants}

et la preuve ne fait (presque) pas intervenir la fonction exponentielle !

L'usage d'un autre dictionnaire (qu'il serait trop long de développer ici) a permis à D. Bertrand (encore !) de tirer du théorème de Yu. Nesterenko des résultats sur les fonctions $\theta$ de Jacobi. Un cas particulier de ses résultats [Ber3] est le suivant :

Si $\alpha \in \mathcal{D}$ est algébrique, les trois nombres $\sum_{n \geq 1} \alpha^{n^{2}}, \sum_{n \geq 1} n^{2} \alpha^{n^{2}}$ et $\sum_{n \geq 1} n^{4} \alpha^{n^{2}}$ sont algébriquement indépendants sur $\mathbb{Q}$. 
Comme dernier corollaire, voici un résultat (parmi d'autres) obtenu par Daniel Duverney, Keiji et Kumiko Nishioka et Iekata Shiokawa [DNNS]: Si $\left\{u_{n}\right\}$ est la suite de Fibonacci $\left(u_{0}=0, u_{1}=1, u_{n+2}=u_{n+1}+u_{n}\right)$, les nombres $\sum_{n \geq 1} u_{n}^{-2}, \sum_{n \geq 1}(-1)^{n} u_{n}^{-2}, \sum_{n \geq 1} u_{2 n-1}^{-1}$ et $\sum_{n \geq 1} n u_{2 n}^{-1}$ sont transcendants.

\section{La preuve de Yu. Nesterenko}

Le but de Yu. Nesterenko est d'appliquer un critère d'indépendance algébrique de Patrice Philippon [Phi1], donc de construire une suite de polynômes $A_{N} \in \mathbb{Z}\left[z, X_{1}, X_{2}, X_{3}\right]$ telle que $\left|A_{N}(q, P(q), Q(q), R(q))\right|$ est petit, avec un contrôle des degrés et des hauteurs (la hauteur $H(P)$ du polynôme $P$ est le maximum des modules de ses coefficients) des $A_{N}$. Il est aussi nécessaire que $A_{N}$ ne s'annule pas dans un voisinage du point

$$
(q, P(q), Q(q), R(q))
$$

ce qui est réalisé si

$$
\left|A_{N}(q, P(q), Q(q), R(q))\right|
$$

n'est pas trop petit. La construction est la suivante :

PREMIER PAS. Une fonction auxiliaire

Pour $N \in \mathbb{N}$ suffisamment grand, le principe des tiroirs (lemme de Siegel) fournit un polynôme $A \in \mathbb{Z}\left[z, X_{1}, X_{2}, X_{3}\right]$ tel que:

les degrés partiels de $A$ sont $\leq N ; \log H(A) \leq 116 N \log N$;

et la fonction $F$ définie par $F(z)=A(z, P(z), Q(z), R(z))$ possède en 0 un zéro d'ordre $M \geq \frac{1}{2} N^{4}$.

Notons que le choix de la majoration des degrés partiels de $A$ par $N$ est assez arbitraire ; nous y reviendrons plus loin. La fonction $F$ n'est pas identiquement nulle car les fonctions $z, P(z), Q(z)$ et $R(z)$ sont algébriquement indépendantes sur $\mathbb{C}([\mathrm{Mah} 2])$, de sorte que $M$ est correctement défini.

La version (classique) du lemme de Siegel utilisée est la suivante :

Lemme de Siegel. Pour $1 \leq i \leq T$ et $1 \leq j \leq S$, soient $g_{i, j} \in \mathbb{Z}$. Si $S>T$, alors il existe des entiers rationnels $x_{1}, \ldots, x_{S}$ non tous nuls tels que $\sum_{1 \leq j \leq S} g_{i, j} x_{j}=0$ pour $1 \leq i \leq T$ et $\max _{1 \leq j \leq S}\left|x_{j}\right| \leq L^{T /(S-T)}$, ò̀ $L=\max \left\{1, \max _{1 \leq i \leq T} \sum_{1 \leq j \leq S}\left|g_{i, j}\right|\right\}$.

La clef de cette étape est le fait que les séries d'Eisenstein ont des coefficients entiers dont la croissance est polynomiale : la borne (mauvaise mais suffisante)

$$
\sigma_{k}(n)=\sum_{d \mid n} d^{k} \leq\left(\sum_{d \mid n} d\right)^{k} \leq n^{2 k}
$$


permet de majorer le module des coefficients de Taylor de

$$
z^{k_{0}} P(z)^{k_{1}} Q(z)^{k_{2}} R(z)^{k_{3}}
$$

par les coefficients de Taylor (de même indice) de $\frac{c^{N}}{(1-z)^{22 N}}$, où $N \geq k_{i}$ et où $c$ est une constante absolue. En effet, la série $1+\sum_{n \geq 1} n^{k} z^{n}$ admet la série majorante $\sum_{n \geq 0} \prod_{1 \leq \ell \leq k}(n+\ell) z^{n}=\frac{k !}{(1-z)^{k+1}}$.

Si $A=\sum_{0 \leq k_{i} \leq N} a\left(k_{0}, \underline{k}\right) z^{k_{0}} \underline{X^{k}} \underline{\underline{k}}$

et $F(z)=A(z, P(z), Q(z), R(z))=\sum_{n \geq 0} b_{n} z^{n}$, les conditions $b_{n}=0$ $\left(0 \leq n<\left[\frac{(N+1)^{4}}{2}\right]\right)$ forment un système linéaire de $\left[(N+1)^{4} / 2\right]$ équations en les $(N+1)^{4}$ inconnues $a\left(k_{0}, \underline{k}\right)$ et les coefficients sont agréablement bornés par le calcul précédent, ce qui permet d'appliquer le lemme de Siegel.

On a ainsi construit une fonction auxiliaire $F(z)=b_{M} z^{M}+\sum_{n>M} b_{n} z^{n}$, où $b_{M}$ est un entier rationnel non nul.

DEUXIÈme PAS. Majoration de $|F(z)|$

Soit $q \in \mathcal{D}$. La majoration des $a\left(k_{0}, \underline{k}\right)$ permet de borner les $\left|b_{n}\right|$ et d'en déduire que

Pour $N$ suffisamment grand et $|z| \leq r=\min \left(\frac{1+|q|}{2}, 2|q|\right)$, on $a|F(z)| \leq|z|^{M} M^{187 N}$.

La condition sur $|z|$ est purement technique ; le fait important est que $|z| \leq r$ avec $|q|<r<1$ et Yu. Nesterenko choisit un tel $r$.

Troisième PAS. Minoration d'un $\left|F^{(T)}(q)\right|$

Il existe un entier naturel $T \leq c_{1}(q) N \log M$ tel que $\left|F^{(T)}(q)\right|>\left(\frac{|q|}{2}\right)^{2 M}$. Cela résulte d'une formule d'interpolation qui n'est autre que la formule des résidus appliquée à

$$
G(z)=z^{-M-1} F(z)\left(\frac{r^{2}-\bar{q} z}{r(z-q)}\right)^{T} .
$$

La clef est la suivante : le module de $F$ est borné par le deuxième pas et, par construction, le résidu en 0 de $G$ est $b_{M}(-r / q)^{T}$, où $\left|b_{M}\right| \geq 1$ car c'est un nombre entier non nul.

QUATRIÈmE PAS. Lemme de zéros 
Lemme de zéros (Nesterenko). Soient $L_{0}$ et $L$ des nombres entiers $\geq 1$. Si $A \in \mathbb{C}\left[z, X_{1}, X_{2}, X_{3}\right]$ est un polynôme non nul de degrés $\leq L_{0}$ en $z$ et $\leq L$ en chacun des $X_{i}$, alors

$$
\operatorname{ord}_{0} A(z, P(z), Q(z), R(z)) \leq 2.10^{45} L_{0} L^{3} \text {. }
$$

Le point crucial qui permet d'obtenir un tel lemme de zéros est le système différentiel satisfait par $P, Q$ et $R$ :

$$
\Theta P=\frac{1}{12}\left(P^{2}-Q\right), \quad \Theta Q=\frac{1}{3}(P Q-R), \quad \Theta R=\frac{1}{2}\left(P R-Q^{2}\right),
$$

mais le $z$ dans $\Theta=\stackrel{\downarrow}{z} \frac{d}{d z}$ est cause d'une (la ?) sérieuse difficulté : il interdit l'usage des lemmes antérieurs de Yu. Nesterenko et oblige à démontrer un résultat qui est loin d'être trivial :

Tout idéal premier non nul de $\mathbb{C}\left[z, X_{1}, X_{2}, X_{3}\right]$ ayant un zéro au point $(0,1,1,1)$ et stable par l'opérateur

$D=z \frac{d}{d z}+\frac{1}{12}\left(X_{1}^{2}-X_{2}\right) \frac{\partial}{\partial X_{1}}+\frac{1}{3}\left(X_{1} X_{2}-X_{3}\right) \frac{\partial}{\partial X_{2}}+\frac{1}{2}\left(X_{1} X_{3}-X_{2}^{2}\right) \frac{\partial}{\partial X_{3}}$ contient le polynôme $z\left(X_{2}^{3}-X_{3}^{2}\right)$.

La conclusion de ce quatrième pas est que dans toutes les inégalités précédentes, on a $M \leq c N^{4}$, ò̀ c est une constante absolue.

Cinquième pas. Qui est $A_{N}$ ?

L'opérateur différentiel $D$ ci-dessus a été étudié pour que, compte tenu du système différentiel satisfait par $P, Q$ et $R$, pour tout polynôme $B \in$ $\mathbb{C}\left[z, X_{1}, X_{2}, X_{3}\right]$, on ait

$$
\frac{d}{d z} B(z, P(z), Q(z), R(z))=\frac{1}{z}(D B)(z, P(z), Q(z), R(z)) .
$$

En particulier, pour $F(z)=A(z, P(z), Q(z), R(z))$, par récurrence sur $t \in \mathbb{N}$, on obtient $z^{t} F^{(t)}(z)=D_{t} A(z, P(z), Q(z), R(z))$, où l'opérateur différentiel $D_{t}$ est défini par $D_{t}=\prod_{0<k<t}(D-k)$.

De plus, il est clair que $12^{t} D_{t} A$ est, comme $A$, un polynôme à coefficients dans $\mathbb{Z}$. Ainsi, avec les notations du troisième pas, il existe $A_{N} \in$ $\mathbb{Z}\left[z, X_{1}, X_{2}, X_{3}\right]$ tel que

$$
(12 z)^{T} F^{(T)}(z)=A_{N}(z, P(z), Q(z), R(z)) .
$$

Enfin, la formule $A_{N}=12^{T} D_{T} A$ permet de majorer le degré $\operatorname{deg} A_{N}$ et la hauteur $H\left(A_{N}\right)$ de $A_{N}$ :

Pour tout entier $N$ suffisamment grand, il existe un polynôme

$$
A_{N} \in \mathbb{Z}\left[z, X_{1}, X_{2}, X_{3}\right]
$$


tel que

$$
\begin{aligned}
\operatorname{deg} A_{N} & \leq c_{2}(q) N \log N, \log H\left(A_{N}\right) \leq c_{2}(q) N(\log N)^{2} \quad \text { et } \\
\exp \left(-\kappa_{2}(q) N^{4}\right) & \leq\left|A_{N}(q, P(q), Q(q), R(q))\right| \leq \exp \left(-\kappa_{1}(q) N^{4}\right),
\end{aligned}
$$

où les constantes $c_{2}$ et $\kappa_{i}$ ne dépendent que de $q$.

Sixième PAS. Conclusion

Un cas particulier du critère d'indépendance algébrique de P. Philippon permet alors de conclure :

CIA (Philippon). Soit $\underline{x} \in \mathbb{C}^{m} ; s^{\prime}$ il existe une suite de polynômes $A_{N} \in \mathbb{Z}[\underline{X}]$ telle que

$$
\begin{aligned}
\operatorname{deg} A_{N} & \leq \sigma(N), \log H\left(A_{N}\right) \leq \sigma(N) \quad \text { et } \\
\exp \left(-\kappa_{2} \lambda(N)\right) & \leq\left|A_{N}(\underline{x})\right| \leq \exp \left(-\kappa_{1} \lambda(N)\right)
\end{aligned}
$$

où les $\kappa_{i}$ sont des constantes positives, $\sigma$ et $\lambda$ sont des fonctions croissant vers l'infini et satisfaisant $\sigma(N+1) / \sigma(N) \longrightarrow 1$ et $\lambda(N) /(\sigma(N))^{k} \longrightarrow \infty$ quand $N \longrightarrow \infty$, alors

$$
\operatorname{degtr}_{\mathbb{Q}} \mathbb{Q}(\underline{x}) \geq k
$$

\section{Mesures et variantes.}

Les estimations du cinquième pas sont assez précises pour que l'on puisse utiliser un critère pour des mesures (critères de Jabbouri, d'Ably ou de Jadot) au lieu du critère d'indépendance algébrique. On obtient ainsi des mesures d'indépendance algébrique et des mesures d'approximation, par exemple :

Soit $q \in \mathcal{D}$, si $\underline{\theta}=\left(\theta_{1}, \theta_{2}, \theta_{3}\right)$ est une base de transcendance de $\mathbb{Q}(q, P(q), Q(q), R(q))$, alors il existe une constante $C>0$ telle que, pour $B \in \mathbb{Z}[X, Y, Z] \backslash\{0\}$, on $a$

$$
\log |B(\underline{\theta})|>-C(t(B)+\operatorname{deg} B \log t(B))^{4}(\log t(B))^{9},
$$

où $t(B)=\max (e, \operatorname{deg} B+\log H(B))$.

Soient $q \in \mathcal{D}$ et $\underline{x}=(q, P(q), Q(q), R(q))$, alors il existe une constante $C>0$ telle que, pour tout point algébrique $\underline{\alpha}=\left(\alpha_{i}\right)_{1 \leq i \leq 4}$, on a

$$
\log \sum_{1 \leq i \leq 4}\left|x_{i}-\alpha_{i}\right|>-C(t(\underline{\alpha}) \operatorname{deg}(\underline{\alpha}))^{4 / 3} \log (t(\underline{\alpha}) \operatorname{deg}(\underline{\alpha})),
$$

où $\operatorname{deg}(\underline{\alpha})=[\mathbb{Q}(\underline{\alpha}): \mathbb{Q}], t(\underline{\alpha})=h(\underline{\alpha})+\log \operatorname{deg}(\underline{\alpha})$ et $h(\underline{\alpha})$ est la hauteur logarithmique absolue de Weil de $\underline{\alpha}$ :

$$
h(\underline{\alpha})=\frac{1}{\operatorname{deg}(\underline{\alpha})} \sum_{v} d_{v} \log ^{+} \max _{1 \leq i \leq 4}\left|\alpha_{i}\right|_{v} .
$$


En fait, la construction précédente ne donne pas exactement ces mesures (l'exposant du facteur correctif en log est moins bon) parce que les paramètres (les degrés partiels du polynôme $A$ du premier pas) ne sont pas choisis de façon optimale : la croissance comparée des fonctions $z$ et $P, Q, R$ permet de donner à $A$ un degré en $z$ plus élevé. Cela se voit bien si l'on utilise l'approche méthodique de $\mathrm{P}$. Philippon (voir [Phi2] et [Phi3]), qui rend très systématiques les preuves de transcendance et d'indépendance algébrique et, de plus, utilise les déterminants d'interpolation de Michel Laurent au lieu de fonctions auxiliaires.

D'autre part, dans son approche méthodique, P. Philippon propose deux autres voies pour conclure à partir de la construction transcendante : dans la première voie, le critère d'indépendance algébrique est remplacé par des propriétés générales d'approximation ; cette voie permet d'atteindre, pour l'instant, les degrés de transcendance 3 ou 4 (ce point de vue n'est pas très éloigné des travaux de Damien Roy et $\mathrm{M}$. Waldschmidt). Dans la deuxième voie le lemme de zéros et le critère sont remplacés par une mesure de transcendance. Pour illustrer ce deuxième type d'idée, voici la preuve donnée par P. Philippon d'un cas particulier (contenant l'indépendance algébrique de $\pi, e^{\pi}$ et $\left.\Gamma(1 / 4)\right)$ du théorème de Yu. Nesterenko :

La construction de transcendance est celle qui a été présentée ci-dessus, mais en un peu plus simple : au troisième pas, il suffit d'avoir $F^{(T)}(q) \neq 0$ et, sans utiliser le lemme de zéros, la conclusion du cinquième pas est

$$
\begin{aligned}
\operatorname{deg} A_{N} & \leq c(q) N \log M, \log H\left(A_{N}\right) \leq c(q) N(\log M)^{2} \quad \text { et } \\
0 & <\left|A_{N}(q, P(q), Q(q), R(q))\right| \leq \exp (-\kappa(q) M) .
\end{aligned}
$$

La mesure d'indépendance qu'on utilise alors est une version quantitative [GPhil] du théorème de G.V. Chudnovsky dont on a parlé plus haut :

Théorème (G. Philibert 1988). Soit $\omega \neq 0$ une période d'une fonction elliptique $\wp$ de Weierstrass, d'invariants algébriques $g_{2}$ et $g_{3}$ et soit $\eta$ la quasi-période associée. Pour tout $\varepsilon>0$, il existe une constante $c(\varepsilon)>0$ telle que, pour tout $B \in \mathbb{Z}[X, Y] \backslash\{0\}$ on a

$$
\left|B\left(\frac{\pi}{\omega}, \frac{\eta}{\omega}\right)\right|>\exp \left(-c(\varepsilon) t(B)^{3+\varepsilon}\right) \text {. }
$$

La mesure annoncée par G.V. Chudnovsky était un peu meilleure que cela et $\mathrm{P}$. Philippon [Phi4] vient d'obtenir le bon résultat : dans l'énoncé précédent on peut remplacer $t(B)^{3+\varepsilon} \operatorname{par}(\operatorname{deg} B)^{2}(\log H(B)+\operatorname{deg} B \log \operatorname{deg} B)$ et $c(\varepsilon)$ par une constante absolue. En particulier, les nombres $\frac{\pi}{\omega}$ et $\frac{\eta}{\omega}$ (et donc $\Gamma(1 / 4) \ldots$ ) ne sont pas des nombres de Liouville.

La manière d'utiliser la mesure de Georges Philibert est expliquée par le Lemme. Soient $\theta_{i}(1 \leq i \leq t)$ et $x_{j}(1 \leq j \leq n)$ des nombres complexes tels que chaque $x_{j}$ est algébrique sur $\mathbb{Q}(\underline{\theta})$. Alors il existe des constantes 
$C$ et $T_{0}$ ayant les propriétés suivantes : si $A \in \mathbb{Z}\left[X_{1}, \ldots, X_{n}\right]$ satisfait à $t(A) \leq T$, où $T \geq T_{0}$, et $A(\underline{x}) \neq 0$, alors il existe un polynôme $B \in$ $\mathbb{Z}\left[Y_{1}, \ldots, Y_{t}\right]$ tel que $t(B) \leq C T$ et $0<|B(\underline{\theta})| \leq e^{C T}|A(\underline{x})|$.

Le polynôme $B$ est obtenu par élimination des $x_{j}$ entre $A$ et les polynômes minimaux des $x_{j} \operatorname{sur} \mathbb{Q}(\underline{\theta})$ (voir [Wal2]).

On peut alors terminer la preuve : on suppose que $g_{2}$ et $g_{3}$ sont algébriques et que $P(q), Q(q)$ et $R(q)$ sont algébriques sur $\mathbb{Q}\left(\frac{\pi}{\omega}, \frac{\eta}{\omega}\right)$; alors, à partir de $A_{N}$ le lemme donne un $B_{N}\left(\frac{\pi}{\omega}, \frac{\eta}{\omega}\right)$ et il est assez facile de voir que, pour $N$ suffisamment grand, $B_{N}$ contredit la mesure de G. Philibert. Le résultat obtenu ainsi est le suivant :

Si $J(q)$ est algébrique, alors $\operatorname{degtr}_{\mathbb{Q}} \mathbb{Q}(q, P(q), Q(q), R(q)) \geq 3$.

Notons enfin que, grâce à un lemme de zéros (voir [GPhi2] et la thèse de $\mathrm{K}$. Barré) pour les fonctions polynomiales en $z$ et $J(z)$, K. Barré obtient ([Bar2]) des mesures d'approximation simultanée $|q-\alpha|+|J(q)-\beta|>\ldots$ qui sont de même qualité que celles que l'on déduit des travaux de Yu. Nesterenko, mais meilleures dans le sens qu'elles séparent les contributions de $\alpha$ et $\beta$. La meilleure version de ce lemme de zéros n'est disponible que dans la thèse de K. Barré, donc il n'est pas inutile de la citer :

Lemme de zéros (GP-KB). Si $P \in \mathbb{C}[X, Y] \backslash\{0\}, \operatorname{deg}_{X} P \leq L_{1}$ et $\operatorname{deg}_{Y} P \leq L_{2}$, on a $\operatorname{ord}_{0} P(T, J(T)) \leq 9 L_{1} L_{2}-L_{2}$ pourvu que $L_{1} L_{2} \geq 1$.

Pour terminer, indiquons seulement que ces résultats sont (étonnamment ?) liés à la conjecture des quatre exponentielles, et renvoyons à l'article [Dia] de Guy Diaz sur ces liens.

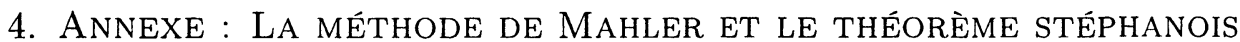

K. Mahler a introduit dans une série de trois articles en 1929 et 1930 une méthode de transcendance que l'on connait sous le nom de méthode de Mahler. Nous allons présenter un cas particulier d'un résultat tiré de [Mah3] et montrer comment la méthode a été modifiée pour parvenir à la preuve du théorème stéphanois.

On considère une fonction $f$ holomorphe au voisinage de zéro, disons sur un disque ouvert de rayon $r+\varepsilon$. On suppose que $f$ est transcendante et que ses coefficients de Taylor à l'origine sont dans $\mathbb{Z}$ (en fait, on pourrait travailler dans un corps de nombres, mais il s'agit ici de présenter le principe de la méthode, donc d'évacuer les problèmes techniques). On suppose aussi qu'il existe une suite de points algébriques $\left(\alpha_{S}\right)_{S \in \mathbb{N}}$ tendant vers 0 et tels que les $f\left(\alpha_{S}\right)$ sont algébriques. On suppose, bien sûr, qu'on a un contrôle des degrés et hauteurs de tous ces nombres algébriques. Le but de la méthode est d'obtenir une contradiction. 
On commence par construire une fonction auxiliaire $F(z)=P(z, f(z))$, où $P$ est un polynôme à coefficients dans $\mathbb{Z}$ de sorte que $F$ ait un zéro d'ordre élevé à l'origine : l'algèbre linéaire fournit l'existence d'un tel polynôme non nul de degrés partiels majorés strictement par $L_{1}$ et $L_{2}$ de sorte que $F$ ait un zéro d'ordre au moins $N$ en 0 dès que $N<L_{1} L_{2}$. Comme $f$ est transcendante, la fonction $F$ n'est pas nulle et, si $M$ est son ordre à l'origine, $z^{-M} F(z)$ tend vers $b_{M} \in \mathbb{Z} \backslash\{0\}$ quand $z$ tend vers 0 .

Le lemme de Schwarz donne alors la majoration $\left|F\left(\alpha_{S}\right)\right| \leq\left|\alpha_{S} / r\right|^{M}|F|_{r}$ (si l'on note $|F|_{r}$ le maximum du module de $F$ sur le disque fermé de rayon $r)$.

Dans l'autre sens on a une minoration arithmétique donnée par l'inégalité de Liouville : si $a_{0} X^{d}+\cdots+a_{d}=a_{0} \prod_{1<j<d}\left(X-\alpha_{j}\right) \in \mathbb{Z}[X]$ est le polynôme minimal du nombre algébrique $\alpha \neq 0$, la mesure de Mahler de $\alpha$ est $M(\alpha)=a_{0} \prod_{1 \leq j \leq d} \max \left(1,\left|\alpha_{j}\right|\right)$ et sa hauteur de Weil est $h(\alpha)=$ $\frac{1}{d} \log M(\alpha)$. Alors l'inégalité de Liouville s'écrit

$$
\log |\alpha| \geq-[\mathbb{Q}(\alpha): \mathbb{Q}] h(\alpha) .
$$

Ici, le nombre $F\left(\alpha_{S}\right)$ est algébrique et il n'est pas nul si $S$ est assez grand car $\alpha_{S}^{-M} F\left(\alpha_{S}\right)$ tend vers $b_{M} \neq 0$ quand $S$ tend vers l'infini. La hauteur de $F\left(\alpha_{S}\right)$ se majore par

$$
h\left(F\left(\alpha_{S}\right)\right) \leq \log L(P)+L_{1} h\left(\alpha_{S}\right)+L_{2} h\left(f\left(\alpha_{S}\right)\right),
$$

où la longueur $L(P)$ est la somme des modules des coefficients de $P$. Si l'on note $\operatorname{deg}(x)$ le degré du nombre algébrique $x$, l'inégalité de Liouville appliquée à $F\left(\alpha_{S}\right)$ donne l'inégalité

$$
\begin{aligned}
M \log \left|\frac{\alpha_{S}}{r}\right| & +\log |F|_{r} \\
& \geq-\operatorname{deg}\left(F\left(\alpha_{S}\right)\right)\left(\log L(P)+L_{1} h\left(\alpha_{S}\right)+L_{2} h\left(f\left(\alpha_{S}\right)\right)\right) .
\end{aligned}
$$

Il s'agit alors d'obtenir une contradiction. Nous allons voir sur un exemple comment procède K. Mahler.

UN BON EXEMPLE La fonction considérée est définie sur le disque unité ouvert par $f(z)=\sum_{n \geq 0} z^{d^{n}}$, où $d$ est un nombre entier $\geq 2$. La fonction $f$ est transcendante car sa série de Taylor est lacunaire. On peut aussi retrouver sa transcendance en utilisant la relation fonctionnelle $f\left(z^{d}\right)=f(z)-z$ (comme on prouve la transcendance d'une fonction périodique prenant une infinité de valeurs). On veut démontrer que $f(\alpha)$ est transcendant si $\alpha$ est algébrique.

On suppose donc $\alpha$ et $f(\alpha)$ algébriques. Si $\alpha_{S}=\alpha^{d^{S}}$, la relation fonctionnelle montre que $f\left(\alpha_{S}\right)$ est algébrique pour tout $S \in \mathbb{N}$. Il est clair que le degré de $\alpha_{S}$ est majoré par celui de $\alpha$ et que le degré de $f\left(\alpha_{S}\right)$ est majoré par le produit des degrés de $\alpha$ et $f(\alpha)$, en raison de la relation fonctionnelle. 
Il en résulte que le degré de $F\left(\alpha_{S}\right)$ est un $O(1)$, où ce $O$ ne dépend pas de la construction de la fonction auxiliaire.

Pour les hauteurs, la croissance est un peu plus rapide : on a $h\left(\alpha_{S}\right)=$ $d^{S} h(\alpha)$ et la relation $f\left(\alpha_{S}\right)=f(\alpha)-\sum_{0 \leq s \leq S-1} \alpha_{s}$, obtenue par itération de l'équation fonctionnelle, donne $h\left(f\left(\alpha_{S}\right)\right) \leq \log (S+1)+h(f(\alpha))+d^{S} h(\alpha)$, $\operatorname{car} \sum_{0 \leq s \leq S-1} d^{s} \leq d^{S}$.

L'inégalité $(*)$ devient donc

$$
M \log \left|\alpha^{d^{S}} / r\right|+\log |F|_{r} \geq-O(1)\left(\log L(P)+L_{1} d^{S}+L_{2} d^{S}\right) .
$$

Lorsque $S$ tend vers l'infini, les deux membres de l'inégalité sont du même ordre de grandeur $d^{S}$ et les termes qui dépendent de la construction de $F$ deviennent négligeables. L'inégalité se réduit ainsi à $-M \geq-O(1)\left(L_{1}+\right.$ $\left.L_{2}\right)$, c'est-à-dire $M \leq\left(L_{1}+L_{2}\right) O(1)$. Or, par construction, on a $N \leq M$ et, par exemple pour $N=L_{1} L_{2}-1$, l'inégalité est contredite par un choix quelconque de $L_{1}$ et $L_{2}$ suffisamment grands.

LE CAS DE L'INVARIANT MODUlAIRE. Si on essaie d'appliquer cette méthode à l'invariant modulaire $J$, la première chose à faire est de le rendre holomorphe à l'origine en le remplaçant par $z J(z)$ (c'est ce qui est fait dans l'article originel [BDGP]. On peut aussi, suivant une suggestion de D. Bertrand, considérer un polynôme en $z$ et $J(z)$ et le multiplier par une puissance convenable de $\Delta=\frac{1}{1728}\left(Q^{3}-R^{2}\right)$ comme dans [Bar2] et [Wal1], [Wal2]). La transcendance de la fonction $J$ s'obtient assez facilement en utilisant ses propriétés de modularité. Ici nous poserons $f(z)=z J(z)$.

On suppose $\alpha$ et $J(\alpha)$ algébriques. Alors, pour $\alpha_{S}=\alpha^{S}, J\left(\alpha_{S}\right)$ est algébrique. En effet on a la relation fonctionnelle $\Phi_{S}\left(J\left(z^{S}\right), J(z)\right)=0$, où $\Phi_{S}$ est le polynôme modulaire. Il s'agit d'un polynôme de $\mathbb{Z}[X, Y]$, symétrique en $X$ et $Y$, unitaire en $X$ et de degrés partiels

$$
\psi(S)=S \prod_{p \mid S}\left(1+\frac{1}{p}\right)
$$

de sorte que $\psi(S)=O(S \log \log S)$. Cette relation fonctionnelle montre que le degré de $J\left(\alpha^{S}\right)$ est un $O(\psi(S))$, et cette borne est en fait la meilleure possible (voir [Ber3]). Cette relation fonctionnelle donne aussi une borne pour la hauteur de $J\left(\alpha^{S}\right)$ (c'est celle qui est utilisée dans [BDGP]), mais l'utilisation de la hauteur de Faltings des courbes elliptiques donne une meilleure majoration : $h\left(J\left(\alpha^{S}\right)\right)=O(\log S)$.

Pour la fonction auxiliaire, on obtient ainsi $\operatorname{deg}\left(F\left(\alpha_{S}\right)\right) \asymp \operatorname{deg}\left(J\left(\alpha_{S}\right)\right)$ et

$$
h\left(F\left(\alpha_{S}\right)\right) \leq \log L(P)+\left(L_{1}+L_{2}\right) h\left(\alpha_{S}\right)+L_{2} h\left(J\left(\alpha_{S}\right)\right)
$$


de sorte que l'inégalité $(*)$ devient

$$
S M+c_{2} \leq O(1) \psi(S)\left(c_{1}+\left(L_{1}+L_{2}\right) S+L_{2} \log S\right),
$$

où $c_{1}$ et $c_{2}$ ne dépendent que de la construction (c'est-à-dire des paramètres $L_{1}, L_{2}$ et $N$, de $r$ et des coefficients de Taylor de $f$ ). Comme $\psi(S)$ croît plus vite que $S$, on ne peut plus faire, comme K. Mahler, tendre $S$ vers l'infini pour obtenir une contradiction.

Les difficultés proviennent à la fois du degré et de la hauteur de $J\left(\alpha^{S}\right)$. Différents auteurs ont modifié la méthode de Mahler dans le but, avoué ou non, de conclure dans le cas présent (voir le livre [Nis] de K. Nishioka). La modification principale consiste à utiliser un lemme de Siegel au lieu de la seule algèbre linéaire pour construire la fonction auxiliaire. Cela permet d'estimer effectivement les constantes $c_{1}$ et $c_{2}$ ci-dessus en fonction de $L_{1}, L_{2}$ et $N$. Il faut ensuite trouver une valeur de $S$ pour laquelle $F\left(\alpha_{S}\right)$ n'est pas nul, avec une estimation de $S$ en fonction des paramètres suffisamment bonne pour que l'inégalité ci-dessus puisse aboutir à une contradiction. Je crois que les prédécesseurs des Stéphanois ont cherché à obtenir des théorèmes trop généraux et que c'est la raison pour laquelle ils ne s'appliquaient pas à l'invariant modulaire. On ne saura jamais ce que pensait faire K. Mahler lui-même, quand il disait en 1972 [Mah4] qu'une majoration de la longueur du polynôme modulaire de la forme $L\left(\Phi_{2^{n}}\right) \leq \exp \left(C 2^{n}\right)$ lui permettrait de démontrer sa conjecture. Cela n'est pas très grave car une telle majoration est fausse, ainsi que l'a montré Paula Cohen [Coh] ... Les points cruciaux de la preuve de [BDGP] sont l'utilisation de la croissance des coefficients de Taylor de $z J(z)$ (c'est encore meilleur par la méthode de D. Bertrand, puisque l'on a vu que la croissance des coefficients de Taylor des séries d'Eisenstein est polynomiale) et la détermination de l'entier $S$ convenable. Ce dernier point résulte simplement de l'application du lemme de Schwarz, alors que, habituellement, l'estimation de ce $S$ se fait par comparaison de la série de Taylor de la fonction auxiliaire $F$ avec une série géométrique, procédé qui fournit ici un $S$ trop grand.

\section{BIBLIOGRAPHIE}

[Bar1] K. Barré, Propriétés de transcendance des séries d'Eisenstein. Séminaire de Théorie des nombres de Paris 1994-1995 (à paraître).

[Bar2] K. Barré, Mesure d'approximation simultanée de $q$ et $J(q)$. J. Number Th. 66 (1997), 102-128.

[BDGP] K. Barré-Sirieix, G. Diaz, F. Gramain et G. Philibert, Une preuve de la conjecture de Mahler-Manin. Invent. Math. 124 (1996), 1-9.

[Ber1] D. Bertrand, Séries d'Eisenstein et transcendance. Bull. Soc. Math. France 104 (1976), 309-321.

[Ber2] D. Bertrand, Fonctions modulaires, courbes de Tate et indépendance algébrique. Séminaire Delange-Pisot-Poitou, Paris, 19ème année (1977/78), exposé 36, 11p.

[Ber3] D. Bertrand, Theta functions and transcendence. Madras Number Theory Symposium 1996, The Ramanujan J. Math. 1 (1997), 339-350. 
[Chu] G.V. Chudnovsky, Contributions to the theory of transcendental numbers. Math. Surveys and Monographs 19, Amer. Math. Soc., (1984), 450p.

[Coh] P. Cohen, On the coefficients of the transformation polynomials for the elliptic modular function. Math. Proc. Camb. Phil. Soc. 95 (1984), 389-402.

[Dia] G. Diaz, La conjecture des quatre exponentielles et les conjectures de D. Bertrand sur la fonction modulaire. J. Théor. Nombres Bordeaux 9 (1997), 229-245.

[DNNS] D. Duverney, K. Nishioka, K. Nishioka and I. Shiokawa, Transcendence of Jacobi's theta series and related results. Number Theory - Diophantine, Computational and Algebraic Aspects, K. Györy, A. Pethö and V.T. Sòs eds, Proc. Conf. Number Theory Eger 1996, de Gruyter, Berlin (à paraître).

[GPhi1] G. Philibert, Une mesure d'indépendance algébrique. Ann. Inst. Fourier (Grenoble), 38 (1988), 85-103.

[GPhi2] G. Philibert, Un lemme de zéros modulaire. J. Number Theory 66 (1997), 306-313.

[Lan1] S. Lang, Elliptic functions. Addison-Wesley, Reading, MA, (1973). seconde édition, GTM 112, Springer-Verlag, New York ..., (1987).

[Lan2] S. Lang, Introduction to Modular Forms. Grundlehren 222, Springer-Verlag, Berlin ... , (1976).

[Mah1] K. Mahler, Remarks on a paper by Wolfgang Schwarz. J. Number Theory 1 (1969), 512-521.

[Mah2] K. Mahler, On algebraic differential equations satisfied by automorphic functions. J. Austral. Math. Soc. 10 (1969), 445-450.

[Mah3] K. Mahler, Arithmetische Eigenschaften der Lösungen einer Klasse von funktional Gleichungen. Math. Ann. 101 (1929), 342-366.

[Mah4] K. Mahler, On the coefficients of the $2^{n}$-th transformation polynomial for $j(\omega)$. Acta Arith. XXI (1972), 89-97.

[Man] Yu.I. Manin, Cyclotomic fields and modular curves. Uspekhi Mat. Nauk 26 (1971), 7-71 [en russe]. trad. angl. : Russian Math. Surveys 26 (1971), 7-78.

[Mas] D.W. Masser, Elliptic Functions and Transcendence. L. N. in Math. 437, SpringerVerlag, Berlin ..., (1975).

[Nes1] Yu.V. Nesterenko, Modular functions and transcendence problems - Un théorème de transcendance sur les fonctions modulaires. C. R. Acad. Sci. Paris, Série I, 322 (1996), 909-914.

[Nes2] Yu.V. Nesterenko, Modular functions and transcendence questions. Math. Sb. 187 (1996), 65-96 [en russe]. trad. angl. : Math. USSR Sb. 187 (1996) 1319-1348.

[Nes3] Yu.V. Nesterenko, On a measure of algebraic independence of values of Ramanujan's functions. Trudy Math. Inst. Steklov, 218, (1997), 299-334. trad. angl. : Proc. Steklov Inst. Math., 218, (1997), 294-331.

[Nis] K. Nishioka, Mahler Functions and Transcendence. L. N. in Math. 1631, SpringerVerlag, Berlin ..., (1996).

[Phi1] P. Philippon, Critères pour l'indépendance algébrique. Inst. Hautes Études Sci. Publ. Math. 64 (1987), 5-52.

[Phi2] P. Philippon, Une approche méthodique pour la transcendance et l'indépendance algébrique de valeurs de fonctions analytiques. J. Number Theory 64 (1997), 291-338.

[Phi3] P. Philippon, Indépendance algébrique et K-fonctions. J. reine angew. Math. 497 (1998), $1-15$.

[Phi4] P. Philippon, Mesures d'approximation de valeurs de fonctions analytiques. Acta Arith. 88 (1999), 113-127.

[Sch] Th. Schneider, Einführung in die transzendenten Zahlen. Springer-Verlag, Berlin ... (1957) ; trad. franç. : Introduction aux nombres transcendants, Gauthier-Villars, Paris, (1959).

[Ser] J.-P. Serre, Cours d'arithmétique. Presses Univ. France, Paris, (1970) ; trad. angl. : A course in arithmetic, GTM 7, Springer-Verlag, New York ..., (1973).

[Wal1] M. Waldschmidt, Sur la nature arithmétique des valeurs de fonctions modulaires. Sém. Bourbaki, 49ème année, (1996/97), $\mathbf{n}^{\circ} 824$; Soc. Math. France, Astérisque 245 (1997), 105-140. 
[Wal2] M. Waldschmidt, Transcendance et indépendance algébrique de valeurs de fonctions modulaires. CNTA5, Carleton 1996 ; Proceedings of the fifth Conference of the Canadian Number Theory Association, éd. : R. Gupta et K. Williams, CRM Proceedings and Lecture Notes (AMS) Vol. 19 (1999), 353-375.

François Gramain

Équipe de Théorie des Nombres

Faculté des Sciences

23, rue du Docteur Paul Michelon

42023 ST ETIENNE CEDEX 2

E-mail : gramain@univ-st-etienne.fr 